\section{Doenças diarréicas em menores de cinco anos no Estado de Pernambuco: prevalência e utilização de serviços de saúde}

\section{Diarrheal disease in children under five years of age in the State of Pernambuco, Brazil: Prevalence and utilization of health services}

\section{Resumo}

Objetivou-se, com um estudo transversal de base populacional de 2.074 crianças menores de cinco anos no Estado de Pernambuco (Região Metropolitana do Recife - RMR; Interior Urbano - IU; Interior Rural - IR), avaliar comparativamente a prevalência das diarréias, bem como suas implicações na produção de consultas ambulatoriais e internações hospitalares. Ademais, a pesquisa propõe-se a estabelecer uma linha de base para avaliar tendências temporais do problema. A amostra, de caráter probabilístico, foi distribuída em 18 municípios do Estado, sendo cinco na RMR e os demais nas zonas rurais e urbanas de 13 municípios do interior (Zona da Mata, Agreste e Sertão). A ocorrência mais elevada $(22,7 \%$ nas últimas duas semanas) e a maior demanda de atendimentos ambulatoriais $(8,6 \%$ nos últimos 03 meses) foram encontradas no IU, enquanto as internações hospitalares, $(4,1 \%$ das crianças pesquisadas num retrospecto de 12 meses) predominavam no IR. No conjunto do Estado de Pernambuco, estimouse que para cada cem casos de diarréia ocorreu uma taxa de 0,44 hospitalizações, com freqüências mais elevadas na RMR. Concluiu-se, tomando como referência os compromissos internacionais assumidos pelo Brasil junto às Nações Unidas (UNICEF 1990) para a década passada, que as diarréias ainda representavam um relevante problema de saúde coletiva em todo Estado de Pernambuco, bem como uma pesada carga de demandas sobre os serviços ambulatoriais e, secundariamente, como demandas hospitalares.

Palavras-chave: Epidemiologia das diarréias. Criança. Assistência médica.

A pesquisa obedeceu às exigências éticas da resolução 196 / 96 do Conselho Nacional de Saúde, sendo aprovada pelo Comitê de Ética da Universidade Federal de Pernambuco. 


\section{Abstract}

The aim of the present cross-sectional population study involving 2,074 children under five years of age in the state of Pernambuco, Brazil (Metropolitan Recife - MR; Urban Inland - UI; Rural Inland RI) was to comparatively assess the prevalence of diarrhea as well as the implications of the disease regarding outpatient appointments and hospitalizations. The study proposed to establish a baseline for assessing temporal trends of the problem. The sample was determined probabilistically and distributed among 18 municipalities throughout the state, five of which were located in MR. The remaining 13 municipalities were located in the state's rural and urban inland areas (plantation region; transition region and semi-arid region). The highest occurrence $(22.7 \%$ in the previous two weeks) and greatest demand placed upon outpatient care $8.6 \%$ in the past 03 months) were found in the UI, whereas hospitalizations in a 12-month retrospective survey $(4.1 \%$ of the children studied) were more prevalent in the RI. Throughout the state of Pernambuco, it was estimated that for every one hundred cases of diarrhea, there was a rate of 0.44 hospitalizations, with greater frequencies in MR. Taking as reference the international commitment Brazil has taken with the United Nations (UNICEF 1990) for the past decade, one may conclude that diarrhea remains a relevant public health problem throughout the entire state of Pernambuco and continues to place a heavy burden primarily upon outpatient clinics and secondarily on hospital services.

Keywords: Epidemiology of diarrhea. Children. Medical care.

\section{Introdução}

As doenças diarréicas configuram um dos principais problemas que afetam a qualidade de vida das crianças nos países em desenvolvimento, implicando numa carga considerável de morbi-mortalidade e, conseqüentemente, importantes demandas para a rede de serviços de saúde ${ }^{1,2}$. Em nível individual e em escala coletiva, a instalação e o curso patogênico das diarréias resultam do efeito sinérgico de uma multiplicidade de fatores econômicos, sociais, culturais e biológicos ${ }^{3}$, podendo, em função da natureza intensidade e interação destes condicionantes, ocorrer e evoluir diferentemente entre as várias regiões e grupos populacionais que constituem ecossistemas distintos entre si e mutáveis, no tempo e no espaço ${ }^{1,4}$. Em razão destas características, o estudo das diarréias e seu impacto nas estatísticas de saúde contribui para retratar o perfil epidemiológico de uma população e o peso de suas demandas efetivas ou potenciais sobre a rede de serviços.

Em âmbito mundial, apesar da redução considerável das taxas de mortalidade infantil e pré-escolar nos últimos 25 anos, as doenças diarréicas ainda persistem como um marcante problema de saúde populacional, representando, em muitos países, a principal causa de morte entre crianças de um a quatro anos 5 .

Assim, Kosek et al, consolidando publicações de diversos países para o período 1992 - 2000, estimaram uma incidência anual de 3,2 casos de diarréias por criança menor de cinco anos ${ }^{7}$. Quanto à mortalidade, estes autores relatam que, em termos absolutos, as doenças diarréicas resultaram em 2,5 milhões de mortes em menores de cinco anos, o que representaria $21 \%$ do total de óbitos registrados nesta faixa etária. Estimativas mais recentes de 2000 - 2003 mostraram que a diarréia estava entre as seis causas que explicavam $73 \%$ das 10,6 milhões de mortes em crianças menores de cinco anos, contribuindo com $18 \%$ destas $^{8}$. Até mesmo nos países 
desenvolvidos, as diarréias ainda representam um importante componente das estatísticas de morbidade e de demanda de serviços de saúde, como no caso dos Estados Unidos, onde Parashar et al descreveram 16.700 hospitalizações em doze meses, em crianças menores de cinco anos, por complicações produzidas por síndromes diarréicas ${ }^{9}$.

No Brasil, um estudo realizado por Benício et al, evidenciou que, nas regiões Norte e Nordeste, ocorreriam entre três e quatro episódios de diarréias anuais, em menores de cinco anos, enquanto no Sul do país a freqüência baixava para $1,4 \operatorname{casos}^{10}$. Em 1996, segundo a Pesquisa Nacional sobre Demografia e Saúde, o registro de diarréias nas duas últimas semanas em crianças com menos de cinco anos foi de $18,0 \%$ para o Nordeste e $13,0 \%$ para o Norte, enquanto nas demais regiões a ocorrência de casos se situava em $10 \%{ }^{11}$. Consolidando dados de pesquisas estaduais realizadas no qüinqüênio 1987 - 1992, o UNICEF estimou que, no Nordeste, $16 \%$ das crianças tinham história de diarréia nas duas últimas semanas, prevalência que se elevava para $21,4 \%$ no Estado de Pernambuco ${ }^{12}$. Na realidade, parece consensual que as doenças diarréicas se comportam de forma diferente entre espaços geográficos e grupos socioeconômicos, ilustrando a geopolítica da desigualdade na distribuição do processo saúde/doença.

Neste artigo objetivou-se estudar o problema das diarréias sob diferentes enfoques (prevalência em nível populacional, demandas ambulatoriais e internações hospitalares) em menores de cinco anos no Estado de Pernambuco. Partindo de uma pesquisa tipo inquérito epidemiológico, que se prestou para a dissertação de mestrado de um dos autores, o estudo possibilita a definição de uma linha de base a partir da qual poderiam ser demarcados os pontos referenciais para o acompanhamento do problema em nível populacional e o seu impacto sobre os serviços de saúde ${ }^{13}$. Ademais assumiria, ainda, uma presumível extensão em termos de validade externa, na medida em que pode refletir uma provável analogia com a situação de outras unidades federativas da Região. Com a introdução recente da vacina contra o rotavírus, é provável que ocorra uma modificação substancial no quadro epidemiológico das diarréias e suas implicações (consultas, hospitalizações e mortes), sendo portanto interessante dispor de um quadro consolidado de informações para responder a esta perspectiva.

\section{Metodologia}

\section{O contexto do estudo}

Na época da coleta dos dados de campo, o Estado de Pernambuco tinha uma população estimada de 7.500 .000 habitantes, dos quais cerca de $40 \%$ localizados na Região Metropolitana do Recife (8 municípios). Estimava-se que $71 \%$ viviam em áreas urbanas. Calculava-se que 660.000 famílias (aproximadamente 2.840.000 pessoas) viviam nos anos finais da década abaixo da linha da pobreza, com uma renda "per capita" abaixo de 25 dólares mensais ${ }^{13}$. As condições de vida da população eram bastante heterogênias, o que se expressava, por exemplo, nos indicadores de mortalidade infantil: 37,7/1000 nascidos vivos no Recife e 137/1000 nascidos vivos no Agreste Meridional e na Mata Úmida.

\section{Procedimentos Metodológicos}

As informações utilizadas neste estudo foram disponibilizadas a partir do banco de dados da segunda pesquisa estadual de saúde e nutrição do Estado de Pernambuco ${ }^{14}$, realizada pelo Departamento de Nutrição da Universidade Federal de Pernambuco e o então Instituto Materno Infantil de Pernambuco (IMIP), apoiado financeiramente pelo Ministério da Saúde, com a mediação do extinto Instituto Nacional de Alimentação e Nutrição (INAN).

Assim, o tamanho amostral foi estimado para representar objetivos nutricionais (hipovitaminose A, anemia e desnutrição 
energético-protéico) e condições de saúde de mães e crianças estreitamente relacionadas com o binômio alimentação/nutrição, a partir de valores referenciais (15\% a $45 \%$ ) que, simultaneamente, atendem aos requisitos estatísticos para representação da prevalência da diarréia em âmbito estadual. A amostra foi constituída por 2.078 das quais $737(35,4 \%)$ provenientes da RMR, $687(33,1 \%)$ do Interior Urbano (IU) e 654 $(31,5 \%)$ do Interior Rural (IR). No caso específico das diarréias, a amostra original sofreu quatro perdas (dados de prevalência) e duas perdas (dados de consultas). Admitindo-se em $20 \%$ a prevalência de diarréia para o Estado e adotando-se um nível de confiança de 95\%, a amostra final estudada permitiu estimar a prevalência de diarréia com erro de estimação não superior a 1,7\%.

O processo de seleção amostral foi desenvolvido em três estágios: a) seleção dos municípios com probabilidade quase proporcional à sua população; b) seleção dos setores censitários em cada município; c) seleção de casas/casos (criança) em cada setor censitário ( 40 a 45 unidades amostrais por setor).

As mães ou responsáveis pelas crianças selecionadas eram entrevistadas em seus domicílios, preenchendo o questionário "morbidade referida": casos de diarréias nos últimos 15 dias, consultas por diarréias nos últimos três meses e internações por diarréias nos últimos 12 meses, juntamente com o registro de outras doenças e suas implicações (consultas/internações). Os entrevistadores selecionados, treinados e supervisionados, foram previamente submetidos a um processo de capacitação de 30 dias. Detalhes adicionais sobre os trabalhos de campo, validação dos questionários e processamento de dados podem ser acessados na publicação conjunta de Batista Filho e Romani (2002).

Definiu-se como diarréia, a partir de anamnese referente às últimas duas semanas, a ocorrência de três ou mais evacuações, de consistência líquida ou semi-líquida, acompanhada ou não de muco ou sangue. Nas crianças amamentadas, o discer- nimento das mães decidia o caráter diarréico ou não, nos casos de apenas três evacuações diárias.

Como consultas ambulatoriais foram consideradas as informações obtidas mediante entrevista com as mães ou responsáveis pelas crianças, em relação à pergunta sobre se o caso examinado tinha se submetido a consulta nos últimos três meses, registrando-se como consulta apenas os procedimentos diagnósticos e terapêuticos realizados por médicos no último trimestre. Para as internações hospitalares, facilmente auto-explicativas, foram consideradas as ocorrências de admissões registradas com permanência igual ou acima de 24 horas, bem como o número de vezes por diarréia e outras patologias auto-referidas (pneumonia, desnutrição e outras), no decurso de 12 meses.

Para expressar os resultados numa única base temporal (ano), as prevalências de diarréias em duas semanas foram multiplicadas por 26 semanas, enquanto a anualização das consultas foi obtida multiplicando por quatro os dados referentes ao retrospecto de 3 meses. Outros critérios de construção de indicadores achamse explicitados na tabela pertinente, como notas explicativas (Tabela 1).

Como áreas geográficas foram especificadas a Região Metropolitana do Recife (RMR) o Interior Urbano (IU) e o Interior Rural (IR). Para fins de decisões estatísticas, a estratificação espacial vale para comparações inter-áreas, não sendo representativas para cada espaço geográfico para o evento hospitalização. As informações sobre mortalidade analisadas na discussão foram obtidas de fontes secundárias, em função do reduzido número de óbitos que poderiam ser disponibilizados a partir dos dados primários das famílias entrevistadas. Nas decisões de hipóteses, adotou-se um nível de significância de $5 \%$.

\section{Resultados}

Para o conjunto amostral de todo o Estado, a ocorrência de diarréias nas últimas 
Tabela 1 - Prevalência de diarréia em crianças menores de cinco anos, no estado de Pernambuco, por estratos geográficos - 1997

Table 1 - Prevalence of diarrhea in children under five years of age in the state of Pernambuco according to geographic divisions - 1997

\begin{tabular}{lccccc}
\hline Estrato geográfico & \multicolumn{2}{c}{ Sim } & \multicolumn{2}{c}{ Total } & Estatísticas \\
& $\mathrm{N}$ & $\%$ & $\mathrm{~N}$ & $\%$ & IC: $95 \%$ \\
\hline RMR & 124 & 16,9 & 735 & 100,0 & $(14,2 \%$ a $19,8 \%)$ \\
IU & 156 & 22,7 & 686 & 100,0 & $(19,6 \%$ a $26,0 \%)$ \\
IR & 130 & 19,9 & 653 & 100,0 & $(16,9 \%$ a $23,2 \%)$ \\
ESTADO & 410 & 19,8 & 2074 & 100,0 & $(18,0 \%$ a $21,5 \%)$ \\
\hline
\end{tabular}

duas semanas foi de $19,8 \%$, o que corresponde, como taxa anualizada (projetada para 52 semanas) a uma freqüência de 3,9 episódios por criança/ano. Em relação aos estratos geográficos, o IU apresentou uma prevalência significativamente mais elevada $(22,7 \%)$ do que a RMR $(16,9 \%)$, assumida como espaço de referência para fins comparativos $(\mathrm{p}=0,005)$.

Em relação às demandas ambulatoriais resultantes de episódios diarréicos, 6,1\% das crianças receberam consultas médicas nos três meses que precederam a pesquisa. Extrapoladas para o período de 12 meses, estes valores correspondiam às estimativas de $24,4 \%$ de atendimentos anuais por conta das diarréias.

Em escala trimestral, a freqüência de diarréias demandando consultas ambulatoriais foi mais elevada no IU $(8,6 \%) \mathrm{e}$ menor no IR (4,7\%). Em termos compara- tivos com outros grupos de doenças, as diarréias figuraram, em ordem decrescente, como o quarto fator de demandas ambulatoriais no Estado e na RMR, e como terceiro no interior urbano e rural, com o grupo IRA/pneumonia e "outras causas" prevalecendo em ordem de freqüência percentual. (Tabela 2)

Conforme se verifica no gráfico 1, 10,5\% das crianças investigadas tinham registro de admissões hospitalares nos últimos 12 meses no Estado de Pernambuco, com $2,7 \%$ das internações sendo atribuídas às doenças diarréicas. Na Região Metropolitana do Recife assinalou-se a maior prevalência de internações $(12,2 \%)$ e, ao mesmo tempo, o menor percentual de casos (1,6\%) hospitalizados por diarréias. Já no Interior Rural foi encontrada a mais elevada participação das diarréias como motivo de internações $(4,1 \%)$, o que represen-

Tabela 2 - Percentual de consultas ambulatoriais realizadas nos últimos três meses em menores de cinco anos, por diferentes causas, segundo a situação do domicílio no estado de Pernambuco -1997 Table 2 - Percentage of outpatient visits in the past three years among children under five years of age for different causes, according to housing situation in the state of Pernambuco-1997

\begin{tabular}{lccccc}
\hline Especificações & Estado & RMR & $\begin{array}{c}\text { Interior } \\
\text { Urbano } \\
(\mathrm{n}=687)\end{array}$ & $\begin{array}{c}\text { Total } \\
\text { Rural } \\
(\mathrm{n}=653)\end{array}$ & $\begin{array}{c}\text { Interior } \\
(\mathrm{n}=1.340)\end{array}$ \\
\hline Diarréia & 6,1 & 5,0 & 8,6 & 4,7 & 6,7 \\
IRA / Pneumonia & 19,5 & 21,3 & 19,5 & 17,5 & 18,5 \\
Rotina & 11,6 & 24,6 & 6,6 & 2,1 & 4,4 \\
Outras Causas & 19,5 & 19,4 & 23,0 & 15,8 & 19,5 \\
\hline
\end{tabular}

$\mathrm{RMR}=$ Região Metropolitana do Recife

IRA = Infecção respiratória aguda

$M R=$ Metropolitan Recife

$A R I=$ Acute Respiratory Infection 
ta $35,3 \%$ de todos os atendimentos hospitalares das crianças pesquisadas neste estrato geográfico.

Na Tabela 3 descreve-se uma seqüência de estimativas relacionando prevalências anualizadas de diarréias, números absolutos e respectivos percentuais de consultas anuais, taxa de hospitalização por crianças e pelo número estimado de casos em um ano. Para o conjunto do Estado de Pernambuco, as 504 consultas médicas, para um total calculado de 10.660 casos anuais de diarréia, resultaram na produção de 4,6 demandas hospitalares por 100 casos de episódios diarréicos, com um valor máximo de 5,8\% na Região Metropolitana do Recife e um mínimo de 3,6\% no interior rural. Em termos de taxas de hospitalização das crianças, as doenças diarréicas apresentaram uma ocorrência mais elevada $(4,1 \%)$ no interior urbano, e mais baixa $(1,6 \%)$ na Região Metropolitana do Recife. As taxas anuais de hospitalizações informadas em entrevistas e construídas com base nas estimativas dos casos de diarréias calculados para doze meses variaram entre $0,62 \%$ na Região Metropolitana do Recife e $0,27 \%$ no interior urbano.

No Gráfico 1, ilustra-se, comparativamente, os resultados do total de internações hospitalares por todas as causas e, especificamente, a contribuição percentual das hospitalizações por conta das diarréias, verificando-se a grande importância relativa desta doença no Interior Rural, onde representou uma participação acima de $35 \%$ de todos os casos de internações.

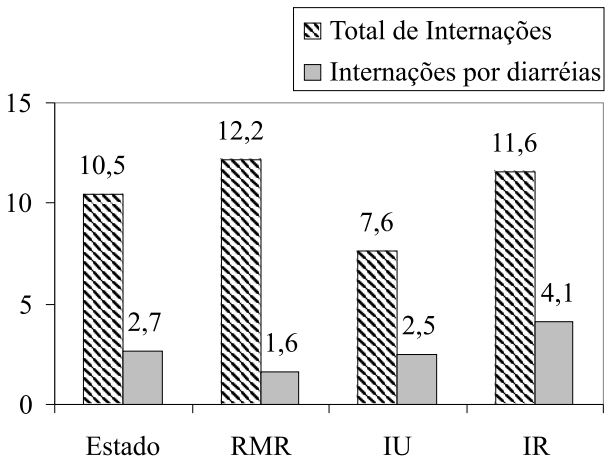

Gráfico 1 - Percentual de internações totais e de admissões hospitalares por diarréias em crianças menores de 5 anos, nos últimos 12 meses, no estado de Pernambuco e seus diferentes estratos geográficos (1997).

Graph 1 - Percentage of total hospitalizations and hospital admissions for diarrhea among children under five years of age in the past 12 months in the state of Pernambuco and different geographic divisions (1997).

Tabela 3 - Casos estimados e taxas anualizadas de consultas médicas e de hospitalização por crianças e por casos de diarréia no Estado de Pernambuco (1997)

Table 3 - Estimated cases and annual rates of medical appointments and hospitalizations children and cases of diarrhea in the state of Pernambuco (1997)

\begin{tabular}{lccccc}
\hline Estrato geográfico & No de casos(a) & \multicolumn{2}{c}{ Consultas anuais (b) } & \multicolumn{2}{c}{ Hospitalizações (\%) } \\
& por ano & No & $\%$ & P / criança (c) & P/ casos(d) \\
\hline Reg. Met. Recife & 3.229 & 144 & $(4,4)$ & $(1,6)$ & $(0,62)$ \\
Interior Urbano & 4.056 & 236 & $(5,8)$ & $(2,5)$ & $(0,27)$ \\
Interior Rural & 3.380 & 124 & $(3,6)$ & $(4,1)$ & $(0,47)$ \\
Estado & 10.660 & 504 & $(4,6)$ & $(2,7)$ & $(0,44)$ \\
\hline
\end{tabular}

(a) Estimativas anualizadas com base na incidência de casos nas duas últimas semanas.

(b) Estimativas a partir da ocorrência de consultas nos últimos 3 meses.

(c) $\mathrm{N}^{\circ}$ de crianças internadas $\times 100 / \mathrm{N}^{\circ}$ de crianças da amostra

(d) № de casos de hospitalização $\times 100$ / No de casos anuais de diarréia.

(a) Annual estimates based on incidence of cases in the past two weeks.

(b) Estimates based on occurrence of appointments in the past three months.

(c) $N^{\circ}$ of children hospitalized $\times 100 / N^{\circ}$ of children from the sample

(d) No of cases of hospitalization $\times 100 / N^{\circ}$ of annual cases of diarrhea. 


\section{Discussão}

Por representar, em escala epidemiológica e como manifestação social, um evento muito ilustrativo das condições de vida da população e das demandas sobre a rede de prestação de serviços, as diarréias podem ser tomadas como um problema que caracteriza, no tempo e no espaço, um estágio do desenvolvimento socioeconômico e, evidentemente, da própria dinâmica do processo saúde/doença. Nesta perspectiva, as diferentes dimensões do problema (prevalência e produção de consultas, internações hospitalares e mortes) retratam aspectos estruturais e conjunturais dos ecossistemas de vida da população. É dentro desta compreensão que o problema é aqui considerado, resgatando-se uma linha de base que, a partir de 1997, pode ser referenciada como um patamar das condições de saúde das crianças no Estado de Pernambuco e, por conseguinte, no acompanhamento posterior de suas tendências, a partir de então. É um enfoque pertinente e oportuno, levando em conta a realização, já assegurada, de um novo inquérito representativo das condições de saúde, nutrição e assistência às mães e crianças no Estado. A análise aqui apresentada distribuise em cinco aspectos:

- o quadro epidemiológico das diarréias;

- as demandas ambulatoriais;

- as internações hospitalares;

- as estatísticas de mortalidade por diarréias, recorrendo-se a dados secundários para todo o Estado e para a Região Metropolitana do Recife; e

- a visão conjunta e integrada destes aspectos.

\section{O quadro epidemiológico das diarréias}

Um dos resultados mais interessantes do estudo consiste na constatação de que as prevalências encontradas para um retrospecto de duas semanas, quando anualizadas (percentual de ocorrências projetado para 52 semanas), implicam em estimativa mínima de 4,4 casos por criança/ano para a RMR e 5,9 casos no IU do es- tado. Estes valores estariam bem abaixo dos dados relatados por Bern et al. para a América Latina, ou seja, 10 ou mais ocorrências por ano em crianças menores de dois anos, o que representaria, portanto, um grupo biológico de maior vulnerabilidade ${ }^{1}$. Mais recentemente, Kosek, Bern e Guerrant, considerando informações de vários países para o período 1992-2000, estimaram a incidência anual de 3,2 casos de diarréia para menores de cinco anos?

Por outro lado, os dados de Pernambuco são bem mais comparáveis com as estimativas de Benício et al., de três a quatro casos anuais para as regiões Norte e Nordeste do Brasil, ou ainda as prevalências relatadas pelo PESMISE em Sergipe ${ }^{15}$ e por Assis e Barreto em Salvador, na Bahia ${ }^{16}$. Em contraste com estes resultados, na Região Sul, Benício et al. descreveram uma ocorrência de 1,4 casos de diarréia criança/ano, em menores de cinco anos, bem semelhante, portanto, à magnitude descrita para os Estados Unidos $(1,1 \%)$. Assim, mesmo no âmbito internacional, os dados de Pernambuco representam um valor bastante elevado, comparando-se aos resultados de estimativas para países africanos e asiáticos (Índia e Bangladesh) ${ }^{10}$.

É provável que os diferenciais de prevalência entre regiões e países pobres e ricos possam explicar também as diferenças entre a Zona Metropolitana do Recife e o Interior Urbano de Pernambuco, com a mediação de variáveis como acesso à água potável, à rede de esgotos, serviços e ações de saúde, nível de escolaridade e de informações dos pais das crianças examinadas, aspectos que serão objeto de um outro artigo. A prevalência de diarréia na Zona Rural, intermediária entre a RMR e o IU, poderia ser atribuída à menor densidade populacional neste espaço, reduzindo os riscos de transmissão mediadas pelo ambiente físico.

\section{As demandas ambulatoriais}

No que diz respeito às demandas ambulatoriais, ressalta-se que, mesmo em 
países adiantados, as diarréias constituem um importante fator de consultas, a exemplo dos Estados Unidos, onde Glass et al. estimaram entre 2.100 .000 a 3.700 .000 casos anuais de consultas pediátricas motivadas por este problema ${ }^{17}$. É evidente que, nos países menos desenvolvidos, as demandas oriundas de diarréias aumentam consideravelmente, retratando a própria carga de doença e sua relação com as condições socioeconômicas e ambientais.

No caso de Pernambuco, a maior procura de assistência médica por conta das diarréias no período de três meses ocorreu em crianças do interior urbano, enquanto a menor freqüência foi registrada na zona rural. É provável que, no último caso, a ocorrência mais baixa de consultas seja resultado de uma demanda parcialmente reprimida, tendo em vista a maior dificuldade de acesso a serviços médicos no meio rural. Ou, ainda, que a ação dos agentes de saúde possa ter resultado na redução do fluxo de casos para consultas médicas. Outra consideração muito pertinente refere-se à análise da composição da demanda por diarréias e outras causas, chamando a atenção, de forma muito relevante, a diferença observada entre o fluxo de "consultas de rotina" na RMR, $(24,6 \%)$ contrastando com $6,6 \%$ no IU e apenas $2,1 \%$ no meio rural. Por diversas razões (econômicas, culturais e condições de acesso) este perfil diferenciado de demanda relativa por itens de atendimento, não se explicando essencialmente por razões epidemiológicas (magnitude da prevalência) expressaria já um aspecto qualitativo do atendimento, de modo que a comparação diarréias x consultas de rotina, com resultados tão discordantes, pode ser interpretada como uma diferenciação induzida pela própria distribuição geográfica dos cuidados de saúde.

\section{As internações hospitalares}

Em princípio, as diarréias nas crianças são processos autolimitados, com uma duração mediana de três dias. Na dependên- cia do agente patogênico, da vulnerabilidade do hospedeiro, mediada por condições inespecíficas como o estado nutricional $^{18,19}$ e por fatores específicos, como os mecanismos imunológicos, bem como procedimentos terapêuticos inadequados, podem se agravar na fase aguda ou evoluir para formas prolongadas, persistentes. Nas duas condições, muitas vezes os processos diarréicos podem demandar os cuidados intensivos da hospitalização.

No caso de Pernambuco, como se observou nos resultados, as diarréias representaram 1/4 de todas as internações de menores de cinco anos no Estado, com uma relação muito baixa na RMR, elevando-se para quase $33 \%$ no IU e alcançando $35,3 \%$ no IR. Assinalou-se, portanto, um gradiente progressivo de admissões hospitalares na medida em que se tornam mais precárias as condições de vida coletiva nos espaços geográficos ${ }^{20}$. Em outras palavras: a partir de uma ocorrência autolimitada, benigna, as diarréias assumem riscos crescentes de agravamento e, portanto, de demandas hospitalares também crescentes. Com estas características de distribuição, as internações por diarréias podem ser interpretadas como indicativas de situações nosográficas bem diferenciadas no espaço territorial, ilustrando a geografia social das doenças.

\section{As estatísticas de mortalidade por diarréias, recorrendo-se a dados secun- dários para todo o Estado e para a Região Metropolitana do Recife}

A mortalidade por diarréias tem caído substancialmente nas duas últimas décadas, seja por conta de fatores de proteção contra sua ocorrência (saneamento ambiental, melhoria das práticas de higiene individuais, familiares e coletivas, aleitamento materno, melhoria do estado nutricional e outros fatores) seja pela massificação de métodos mais adequados para seu manejo terapêutico, como a terapia de reidratação oral (TRO) e os cuidados dietéticos dos casos. Segundo Victora, a mortalidade por di- 
arréia foi a que apresentou maior declínio entre as décadas de 80 e 90 . Assim, de 17,3\% de todas as mortes em 1985-87, as diarréias passaram a ser responsáveis por apenas $8,1 \%$ após 10 anos. No entanto, no Nordeste brasileiro, apesar desta grande redução, continua sendo quase 9 vezes superior a do Sul do Brasil ${ }^{21}$.

Para a década de 90, as metas pactuadas pelas Nações Unidas na Reunião de Cúpula de Nova York preconizavam uma redução de prevalência de $25 \%$ na prevalência e de $50 \%$ na mortalidade, em função da experiência internacional acumulada, demonstrando que é mais fácil tratar os casos do que evitar sua ocorrência ${ }^{22}$. Em comparação com estas metas, observa-se que, no Estado de Pernambuco, a redução de mortalidade por diarréias nas crianças, entre 1990 e 2002, foi de $70 \%$, alcançando $90 \%$ na Região Metropolitana do $\operatorname{Recife}^{23}$, bem acima, portanto, das metas projetadas pelas Nações Unidas.

É muito provável que a redução marcante da mortalidade por diarréia entre as crianças possa ser explicada, de forma específica, pela expansão da TRO e, de forma genérica, pela ampliação do Programa de Agentes Comunitários de Saúde (PACS), Pastoral da Criança e, mais recentemente, o Programa de Saúde da Família (PSF), que produziu impactos substanciais nos indicadores de morbimortalidade ${ }^{24}$.

\section{A visão conjunta e integrada desses aspectos}

Considerando, de forma integrada, os vários aspectos englobados nos resultados e sua análise, torna-se possível estabelecer algumas observações conclusivas ou projetar hipóteses explicativas que demandariam novos estudos.

Assim, parece que os níveis de prevalência do problema não sofreram mudanças substanciais, seja no que se refere à comparação com o cenário mundial na década analisada por Kosek et al. (2003), seja em relação ao quadro regional retratado por Benício (1989), ou, ainda, o relatório do UNICEF(1995), consolidando resultados estaduais publicados entre 1987 e 1992. No entanto, o curso patogênico, em termos de ocorrência de casos graves, parece ter se modificado muito positivamente, já que para 100 ocorrências o número de consultas médicas se situaria em $4,6 \%$, sem grandes variações para o espaço territorial. Esta é a previsão universal para as diarréias: mais de 95\% dos casos devem evoluir como episódios autolimitados. Supõe-se, portanto, que as mães, melhor informadas, ao lado dos agentes comunitários de saúde, estejam mais capacitadas para o manejo bem sucedido do problema, evitando as complicações que resultam em consultas médicas. No meio rural, no entanto, onde aparecem os percentuais mais baixos de consultas, as taxas de hospitalização $(4,1 \%)$ chegam a exceder os próprios percentuais de consultas ambulatoriais, indicando, portanto, que estes casos seriam predominantemente tratados em hospitais, o que representaria sem dúvida uma distorção do modelo esperado de prestação de cuidados de saúde.

No contexto destas análises é importante considerar o declínio marcante e até inesperado da mortalidade de crianças por diarréia: $70 \%$ para o Estado e $90 \%$ para o Recife. Desde que o quadro epidemiológico (prevalência) não se alterou substantivamente, seria bem aceitável supor que os resultados positivos em termos de mortalidade ocorreram pela socialização de condutas mais apropriadas entre usuários e comunidades, a exemplo da TRO, do que as próprias consultas médicas, e, por fim, que o manejo dos casos de internação tenham se tornado mais eficazes. Se esta perspectiva for de fato realista, a alternativa mais imediata para o encaminhamento do problema das diarréias consistiria em atuar sobre o quadro epidemiológico, reduzindo seus fatores mais evidentes de risco. A vacinação contra o rotavírus, já iniciada em Pernambuco, seria um passo importante e imediato neste sentido ${ }^{6}$.

Deve-se, por fim, considerar que grande parte dos resultados expostos na Tabela 3 foram obtidos a partir de soluções matemáticas, como a anualização de inci- 
dência de casos de diarréias a partir dos dados de ocorrência de duas semanas ou da projeção de consultas médicas anuais com base nas demandas de três meses. $\mathrm{Na}$ realidade, as diarréias podem apresentar um comportamento sazonal que dificilmente pode ser apreendido num estudo de caráter seccional, como o que produziu o banco de dados aqui analisado. A World Health Organization (WHO $)^{25}$ destaca estes aspectos, a partir da própria confecção dos questionários. Isto, evidentemente, influiria também no número de consultas médicas. No entanto, com as limitações impostas pelo arquivo da pesquisa, não sobra outro encaminhamento imediato para solucionar esta circunstância, que inclusive, torna-se de controle mais difícil considerando o período de 4 meses de estudo (fevereiro a maio) e dos desencontros de estações (basicamente, verão e inverno) nas diversas áreas fisiográficas do Estado (Litoral /Mata, Agreste e Sertão). Por fim, não se dispõe de dados sobre a mortalidade por diarréias que, a partir de fontes secundárias, pudesse medir, de forma desagregada para os municípios e setores censitários pesquisados, as estatísticas de mortes por diarréia para o ano de 1997, daí o recurso de se utilizar, como referência, as informações globais para o Estado e para o chamado Grande Recife. Estas limitações, no entanto não chegariam a comprometer os objetivos do estudo.

\section{Referências}

1. Bern C, Martinez J, Zoysa I, Glass RI. Magnitude del problema global de las enfermidades diarréicas: actualizacion decenal. Bol Ofic Sanit Panam 1993; 115: 523-35.

2. UNICEF.(Fundo das Nações Unidas para a Infância) Situação mundial da infância. Brasília (DF); 2005.

3. Rouquayrol MZ, Goldbaum M. Epidemiologia, história natural e prevenção de doenças. In: Rouquayrol MZ, Almeida Filho N. Epidemiologia e saúde. Rio de Janeiro: Medsi; 2003. p. 17-35.

4. Benício MHDA, Monteiro CA. Tendência secular da doença diarréica na infância na cidade de São Paulo (1984 - 1996). Rev de Saúde Pública 2000; 34: 83-90.

5. Yunes J, Díaz A. A situação da saúde materno-infantil e suas tendências na América Latina e no Caribe. In: Benguigui Y, Land S, Paganini JM, Yunes J. Ações de saúde materno - infantil a nível local: segundo as metas da cúpula mundial em favor da infância. Washington (DC): OPAS; 1997. p. 3-16.

6. Ministério da Saúde. Doença diarréica por rotavírus: vigilância epidemiológica e prevenção pela vacina oral de rotavírus. Brasília (DF); 2005.

7. Kosek M, Bern C, Guerrant RL. The global burden of diarrhoeal disease, as estimated from studies published between 1992 and 2000. Bull World Health Organ 2003; 81: 197-203.

8. Bryce J, Boschi-Pinto C, Shibuya K, Black RE. Who estimates of the causes of death in children. Lancet 2005; 365: 1147-54.

9. Parashar UD, Breese JS, Glass RI. The global burden of diarrhoeal disease in children. Bull World Health Organ 2003; 81. 236.
10. Benício MUA, Cesar CLG, Gouveia NG. Perfil de morbidade e padrão de utilização de serviços de saúde das crianças brasileiras menores de cinco anos, 1989. In: Monteiro MFG, Cervini R. Perfil estatístico de crianças e mães no Brasil. Rio de Janeiro : IBGE, UNICEF; 1992. p. 79-95.

11. BEMFAM (Sociedade Civil Bem Estar Familiar no Brasil). Pesquisa Nacional sobre Demografia e Saúde. Rio de Janeiro; 1996.

12. UNICEF (Fundo das Nações Unidas para Infância). Saúde e Nutrição das Crianças Nordestinas. Pesquisas Estaduais 1987-1982. Brasília(DF); 1995.

13. Vasconcelos MJOB. Prevalência, demandas ambulatoriais e hospitalização por diarréia em crianças, segundo variáveis nutricionais e fatores sócio-ambientais no Estado de Pernambuco [dissertação de mestrado]. Recife: Departamento de Nutrição da Universidade Federal de Pernambuco; 2006.

14. Batista Filho M, Romani SAM. Alimentação, nutrição e saúde no Estado de Pernambuco. Recife: IMIP; 2002.

15. Sergipe. Secretaria da Saúde. III Pesquisa de Saúde Materno Infantil e Nutrição do Estado de Sergipe PESMISE/98. Aracajú; 2001.

16. Assis AM, Barreto ML. Condições de vida, saúde e nutrição na infância em Salvador. Salvador: Escola Nutrição da Universidade Federal da Bahia; 2000.

17. Glass RI, Lew JF, Gangarosa RE, Lebaron CW, Hom S. Estimates of morbidity and mortality rates for diarrheal diseases in American children. J Pediatr 1991; 118: 27-33.

18. Biesel WR. Metabolic response of the host to infections. In: Feigin RD, Cherry JD. Pediatrics infections disease. Philadelphia: Saunders; 1992. 
19. D'Avila EMM. Estudo epidemiológico de alguns problemas nutricionais. In: Gouveia ELC. Nutrição Saúde e comunidade. Rio de Janeiro: Revinter; 1999. p. 126.

20. Vergolino JRO, Monteiro Neto A. A economia de Pernambuco no limiar do século XXI. Recife: Bagaço; 2001.

21. Victora CG. Intervenções para reduzir a mortalidade infantil pré - escolar e materna no Brasil. Rev Bras Epidemiologia 2001; 4: 3-69.

22. UNICEF (Fundo das Nações Unidas para Infância). Estratégias para melhorar a nutrição de crianças e mulheres nos países em desenvolvimento: um exame de políticas. New York; 1990.
23. Ministério da Saúde. Informações de saúde. Disponível em http:/ / www.datasus.gov.br. [Acessado em 20 de janeiro de 2005].

24. Ministério da Saúde. Saúde da família no Brasil: uma análise de indicadores selecionados: 1998 -2004. Brasília (DF); 2006. (Série C. Projetos, Programas e Relatórios).

25. World Health Organization; Division for Control of Diarrhoeal and Acute Respiratory Disease. Household survey manual: diarrhoea and acute respiratory infections. Geneva; 1994.

Recebido em: 19/09/06 Versão final reapresentada em: 05/11/07 Aprovado em: 04/12/07 